\title{
African Americans Healthcare Disparities and Inclusive Practice; A Literature Review
}

\author{
Shayna S Perdue RN, BSN, FNP(s) and Dr. Abigail Mitchell RN, MSN, MBA \\ D’Youville College, Buffalo NY, USA
}

*Corresponding author: Abigail Mitchell RN, D’Youville College, Buffalo NY, USA, Email: mitchela@dyc.edu

\section{Review Article \\ Volume 4 Issue 1}

Received Date: December 15, 2020

Published Date: January 05, 2021

DOI: $10.23880 /$ jqhe-16000199

\section{Abstract}

African American culture is both fascinating and immense. Unfortunately, there are many disproportions faced by African Americans, which result in barriers to care. There have been a few themes identified in the literature. The themes include: Barriers to care for women, men, children, and then disease specific. Only around $10 \%$ of African Americans receive referrals to a health professional for assistance. This literature review provides information on each theme but also the importance of being culturally competent to provide positive outcomes for this population as health care providers.

African American culture is both fascinating and immense. Unfortunately, there are many disproportions faced by African Americans, which result in barriers to care. For example, there is no solid percentage of African Americans diagnosed with depression; however a prevalence rate is thought to be around $9 \%$ to $13 \%$ of the population. Only around $10 \%$ of African Americans receive referrals to a health professional for assistance, and around $40 \%$ choose to seek assistance from their clergy.

Keywords: American Health Care; Nursing; Africans; Patients

\section{Underrepresentation of African Americans in Nursing}

The inclusion of African Americans in various pursuits continues to be a struggle. This is especially true in the nursing profession. African Americans only compose $10.7 \%$ of nurses with a baccalaureate degree, compared to Non-Hispanics counterparts that compose $69.3 \%$ of baccalaureate nursing degrees. Jean Baptiste, et al. [1] conducted a study among 16 African American women that graduated between 2010 to 2015 to study enrollment and retention among African Americans in BSN programs. Participants felt as though inner strengths, sacrificing, being inclusive, receiving support from others and being receptive assisted in their success [1]. Church and prayer were also mentioned by participants as a supportive factor. Financial needs are another important factor in determining success and being capable of attending and completing BSN programs. According to Jean Baptiste, et al. [1] "Increasing the number of African Americans among health caregivers will increase trust and participation in research studies for health issues that affect African Americans (pp: 48). Cultural competency is another important factor among clinicians and professionals.

The African American population continues to rise, making the need for inclusion vital [2]. To assess knowledge of African American diversity, Odhiambo, et al. [2], conducted a study among nine registered nurses. The sample size consisted of all women, from varying backgrounds, and one of the registered nurses among participants identified as African American [2]. Eight of the nine nurses reported a deficient knowledge of African American culture, and stated they had minimal dealings with African Americans [2]. The 
nurses stated when they provided care to patients, they attempted to treat them fairly, but some nurses felt racism was a thing of the past. One participant from the study explained that race was not often discussed in her family, and she planned to keep discussion of it out of her professional practice as well [2]. Nurses reported in the study that mainly staffing on their floors consisted of Caucasian staff, while one nursing unit was predominantly Asian, and a presence of African Americans nurses was scant. With the growing population, and increasing number of minorities, it is crucial to continue to be inclusive and represent different ethnicities in the public sector.

\section{Issues Among African American Women}

African American women face multiple challenges regarding mental and physical health. There often is a strong bond between African American mothers and their daughters. African American mothers pass down food preparation techniques, views on health/wellness, society, and other traditions [3]. In turn, daughters then pass this down to their daughters. Healthy and unhealthy patterns, as well as exercise routines are mirrored between mother and daughter, since many African American daughters view their mothers as role models. Often, this bond continues, and African American daughters become caregivers to their ailing mothers later in life.

African American daughters were interviewed in a study conducted by Cannon, et al. [4]. The women provided care to their mothers, age 65 and older, and participants were recruited from local churches and organizations. Participants were given basic interview schedules to record their perceptions on their caregiving role and the stressors they experienced. Many of the daughters that partook in the study were employed, college educated, and had average incomes of around 50,000 dollars. Higher instances of mental health issues were found among the caregiver daughters, which included depression and anxiety. These findings tend to be higher in African Americans versus Caucasians.

Health disparities and stressors continue to be found among African American women. Contributing causes include obesity, which can lead to both physical and mental health complications [5]. A study was conducted over a 24-week period involving African American women that had an unhealthy BMI of or equal to 30 , as well as a waist circumference of greater than 35 inches. To improve health outcomes, the women (aged between 19-64), participated in two weekly sessions for a total of 12 weeks. The sessions included exercise, health education on healthier food choices, and socialization among participants. After the 12 weeks, participants were then to continue exercising and choosing healthy food choices on their own using materials provided, as well as using prayer and meditation. Only 55 of the 106 participants completed the program. Medical conditions found among the African American women included hypertension, diabetes, and depression. Depression findings were found to be decreased while socializing with the other women (at the start of the study) and increased as the women continued on their own. This shows there is a correlation between mental health, socialization, and weight loss.

Pregnant African American women are even more susceptible to health detriments and stress, as evidenced by a study conducted by Jallo, et al. [6]. Pregnant women were provided with guided imagery materials to listen to, which included a CD player, CD's, and batteries. The women were to record their experiences while listening to the relaxation CD's and then they were interviewed to express their feelings. Common themes found among the women were ample amounts of stress in their lives, such as financial, environmental, and even relationship strains. The women reported feeling relaxed while listening to the CD's. However, they also reported being interrupted, issues with the equipment, and even having a hard time focusing due to ongoing stressors. Pregnancy can be a stressful time for many women, unfortunately, African American women experience higher levels of stressful events versus any other ethnicity. Improvements to decrease disparities among African American women involve cultural sensitivity, inclusion, and holistic treatment to decrease stressors and facilitate coping mechanisms.

\section{Issues Among African American Men}

African American men are subjected to higher rates of unfavorable social, financial, and racial inequalities that have detrimental effects on their overall well- being [7]. Barriers also exist for African American males when seeking healthcare and lead to decreased compliance [8]. Often, independence and self-sufficiency lead African American males to delay seeking treatment in fear of social oppression. Depression found among African American men also prevents them from seeking care, and some men view these tendencies as protective and positive factors for themselves that show they are strong. This confirms many African American men do not like to feel as though they are not capable or weak, compared to common stereotypes.

Among issues associated with seeking care, many African American men reside outside of their children's homes. This can also lead to further mental health issues and was studied by Tsuchiya, et al. [7]. Participants completed surveys to assess levels of depression, perceived 
neighborhood demographics, interpersonal stress, as well as social supports among the Black fathers. Moderate levels of stress were found among the men, some social supports were present within their lives, and high levels of depression were reported. Financial issues were a major theme that contributed to depression, but neighborhood demographics did not influence depression among the fathers. Social factors assisted in decreasing depression, as well as involvement with their children.

African American men partaking in the study reported increased levels of involvement with their children, despite physical and mental barriers. This is important, since fathers play a vital role in the development of their children [9]. African American fathers, just as fathers of other races are very present and active in their children's lives, despite how this is depicted in society. Racism can negatively portray and minimize the active role of the African American father. Research and studies show that some African American fathers partake in daily care of their children even more so than other ethnicities.

\section{African American Children}

Attention-deficit hyperactivity disorder (ADHD) has been found to impact African Americans youth. As ADHD diagnoses rise, ADHD rates have risen from 5.5\% to $9.6 \%$ between the span of 2011 through 2013 alone [10]. Research studies among African Americans and ADHD is very limited, and racial disproportions have been found. Even though diagnosis rates are increasing among African Americans, Caucasian children receive a diagnosis more than African American children, showing that many are going untreated. Once again, stigma plays a large role in African Americans seeking care for symptoms; parents are reluctant to employ medications in the treatment of their children and are often fearful of labels on their [10]. Mindful consideration and cultural sensitivity to these apprehensions must be considered. Educating, answering questions, and being straightforward with African Americans to provide them with what to expect during treatment and testing may encourage them to comply with treatment and medications.

Disparities also exist among African American youth while involved in foster care, and when exiting or aging out of the foster care system [11]. It has been shown that higher rates of mental health issues have been found among those involved in the foster care system. It is thought when foster care youth age out of the system they will be able to care for themselves mentally and physically. However, this is often not the case and increases their chances of homelessness, inability to find jobs, a decrease in education, and even criminal involvement.

\section{African Americans Living with HIV}

Depression is commonly found among those living with HIV and can decrease quality of life for those impacted. A study conducted by Coleman, et al. [12] included 70 African American men and women that are seropositive. Twentyminute surveys were completed by the participants who assessed depression, pain, social interactions, and current health states. The study included men and women between the ages of 19 through 54, and participants presented with varying sexual preferences, such as heterosexual, gay, and lesbian. Of the participants, $43 \%$ stated that they were involuntarily made to engage in sexual activities at least one time in their lifetime.

More than $50 \%$ of participants presented with symptoms of depression, $44 \%$ did agree to receive treatment, and $34 \%$ were hospitalized due to their depression symptoms. According to Peltzer, et al. [13], "Although African Americans represent $13 \%$ of the female population in the United States, $64 \%$ of U.S. females who are HIV-positive are African American women" (p. 111). Religion and engaging in proper care for one's self has shown to be beneficial and improve health outcomes. Peltzer, et al. [13] also showed that positive social supports increased mood and health in HIV positive women, while stigma remains a large barrier for HIV patients. Peltzer, et al. [13] conducted a literature review to find common themes among women living with HIV, which included faith-based practice, and family supports to cope.

Peltzer, et al. [13] conducted a study that included 11 HIV infected African American women, and they were interviewed on their experiences of living with HIV. A second interview was conducted to obtain further explanation on their responses. Many of the women reported no longer feeling normal, stated their mental health was impacted, and that they often felt degraded by health professionals [13]. Each woman involved in the study stated they felt depressed, showing depression as a very common theme. HIV awareness and education are important among healthcare workers and patients to deliver empathic/self-aware, non-judgmental, and holistic care to all patients.

\section{Mental Health Disparities among African Americans}

Many issues contribute to inequality among African Americans. However, African Americans continue to try to overcome barriers using their mentioned protective factors. During a study, twenty-eight African Americans experiencing mortgage strain were interviewed. The participants resided in Locust Park, where $80 \%$ of the residents are homeowners [14]. The participants contained mainly female participants, 
many over the age of 50 , and they were either employed or on disability. Mortgage strain when described by the participants seemed to threaten pride and their feelings of stability and independence.

Many participants stated they feared the stigma associated with seeking help or social supports. As a result, many participants reported depression, and even feelings of committing suicide. Only a small percentage of participants sought help and instead suppressed these feelings further. This study reveals how stigma can truly hinder treatment. Some African Americans truly perceive medical treatment or assistance as a weakness and it will prevent them from seeking much needed care.

There are numerous mental health diagnoses found among African Americans, but there is question on the validity of over and underdiagnosing. African Americans are often diagnosed with Schizophrenia spectrum disorders, even more so than Caucasian Americans [15]. One cause by researchers is thought to be based on their reporting of spirituality practices. In a study by Peltier, et al. [15] 522 patients were screened in a study, with 471 participants identifying as African American. The participants were screened utilizing interviews to assess for auditory and visual hallucinations that are present in Schizophrenia spectrum disorders, or if religious practices or beliefs persuaded their accounts.

Through additional follow up on the reports of participants, $63.4 \%$ participants provided a spiritual rational, $15.1 \%$ declared to have visual and auditory hallucinations, while $5.3 \%$ of these hallucinations were found as a diagnostic impression of Schizophrenia spectrum disorders. Misappropriation and decreased exploration of symptoms can lead to misdiagnosis, as well as failing to identify other medical causes or even substance abuse. It is important to convey a comfortable and safe setting for African American patients to express and explain their concerns. Clinicians must utilize follow up questions and open communication to address the needs of these patients.

Screening for mental health disorders is not only important, but vital to formulating diagnoses, as well as initiating proper and prompt treatment. Often, primary care providers may be the only chance to assist with mental health disorders such as depression, and can identify and set the tone of treatment sought by African Americans. Randle, et al. [16] conducted a study by reviewing electronic medical records of patients ages 19 and over, from two medical clinics. The urban and rural clinics newly employed the Patient Health Questionnaire-9, a screening tool with 9 questions to assess for depression. Prior to this implementation, the clinics did not have an assessment or tool in place to evaluate depression amongst patients.

\section{The Role of the African American Church in Mental Health}

The African American church plays a vital role in the lives of many African Americans. The church is often viewed as a safe haven and culturally identifiable to many African Americans. The church's credibility also serves as a go to for financial, physical, spiritual, and mental health needs [17]. A study conducted by Anthony, et al. [18] formulated a workshop for African American clergy to increase their knowledge on depression. Prior to the initiation of the workshop, the clergy member's knowledge level was assessed. The study utilized a 5-point Likert scale to gather a baseline on their comprehension of depression, treatment modalities, and their perceptions of depression. There were 42 participants total, which included 19 men and 23 women. After educating the clergy during the workshop, three months later, their knowledge was reassessed with the same questions.

The men and women involved in the study proved they retained knowledge taught to them, as well as learned new knowledge. Clergy members were able to identify the type of professionals able to treat depression, causes of depression, and ways depression can be treated. The clergy felt as though their knowledge improved, and they expressed feeling better prepared to assist their members and refer them for services. It is important that clergy members feel equipped and trained to assist members and the community with mental health issues, since they are often the first and only source of health for many African Americans.

\section{Conclusion}

Findings from the literature review reveal that a lack of knowledge exists in nursing and patient care practice regarding African Americans. African Americans are inundated with barriers in seeking health care. Stigma, knowledge deficits, and a decreased access to care increase these limitations. Numerous studies identify distrust of researchers, health care providers, and various organizations within the United States [19]. Alarmingly, African Americans only comprise $10.7 \%$ of nurses with a baccalaureate degree, compared to Non-Hispanics counterparts that compose $69.3 \%$ of baccalaureate nursing degrees. Enhancements in diversity will not only facilitate trust but will encourage more patients to seek care within their communities $[20,21]$. Utilizing positive and trusted resources within the community, such as the African American church, also assists to bridge gaps in care. The need for healthcare providers to empathize, educate, and support African American patients is crucial. 


\section{References}

1. Jean Baptiste AM (2019) Recruiting and Retaining African Americans in BSN Programs. ABNF Journal 30(2): 44-49.

2. Odhiambo B, Murray Garcia J, Ackerman Barger K (2019) Exploring Nursing Knowledge of Black American Diversity. Journal of Cultural Diversity 26(2): 69-75.

3. Dobal MT (2019) African American Mothers' Influence on Their Daughters' Food and Activity Preferences, Decisions, and Behaviors. ABNF Journal 30(1): 16-21.

4. Cannon S, Fawcett J (2018) Correlates of Psychological and Physical Health Outcomes among African American Caregiving Daughters. ABNF Journal 29(3): 86-97.

5. Groh CJ, Urbancic JC (2015) The Impact of a Lifestyle Change Program on the Mental Health of Obese UnderServed African American Women. Archives of Psychiatric Nursing 29(2): 76-82.

6. Jallo N, Salyer J, Ruiz RJ, French E (2015) Perceptions of Guided Imagery for Stress Management in Pregnant African American Women. Archives of Psychiatric Nursing 29(4): 249-254.

7. Tsuchiya K, Qian Y, Thomas A, Hill De Loney E, Caldwell CH (2018) The Effects of Multiple Dimensions of Risk and Protective Factors on Depressive Symptoms Among Nonresident African American Fathers. American Journal of Community Psychology 62(3/4): 464-475.

8. Powell W, Adams LB, Cole Lewis Y, Agyemang A, Upton RD (2016) Masculinity and Race-Related Factors as Barriers to Health Help-Seeking Among African American Men. Behavioral Medicine 42(3): 150-163.

9. Revell MA (2015) The African American Father Does Matter in Parenting. International Journal of Childbirth Education 30(1): 25-29.

10. Mattox GA, Vinson SY (2018) Culturally Competent Approaches to ADHD: Issues in African-American Populations. Psychiatric Times 35(9): 19-20.

11. Villagrana M (2017) Racial/Ethnic Disparities in Mental
Health Service Use for Older Foster Youth and Foster Care Alumni. Child \& Adolescent Social Work Journal 34(5): 419-429.

12. Coleman CL (2017) Health Related Quality of Life and Depressive Symptoms among Seropositive African Americans. Applied Nursing Research 33: 138-141.

13. Peltzer J, Domian E, Teel C (2015) Living in the Everydayness of HIV Infection: Experiences of Young African-American Women. MEDSURG Nursing 24(2): 111-118.

14. Keene DE, Cowan SK, Castro Baker A (2015) RESEARCH AND PRACTICE. "When You're in a Crisis Like That, You Don't Want People to Know": Mortgage Strain, Stigma, and Mental Health. American Journal of Public Health 105(5): 1008-1012.

15. Peltier MR, Cosgrove SJ, Ohayagha K, Crapanzano KA, Jones GN (2017) Do they see dead people? Cultural factors and sensitivity in screening for schizophrenia spectrum disorders. Ethnicity \& Health 22(2): 119-129.

16. Randle AC, Spurlock AL, Kelley S (2019) Depression Screening Among African American Adults in the Primary Care Setting. Journal of Psychosocial Nursing \& Mental Health Services 57(10): 18-23.

17. Brand DJ (2017) The African American Church: A Change Agent for Health. ABNF Journal 28(4): 109-113.

18. Anthony JS, Morris E, Collins CW, Watson A, Williams JE, et al. (2016) Equipping African American Clergy to Recognize Depression. Journal of Christian Nursing 33(4): 230-237.

19. Reifenstein K (2018) A Commentary: Will We Ever Get Enough? Strategies to Enhance Minority Participation in Research. ABNF Journal 29(1): 17-26.

20. Alang S, McAlpine D, McCreedy E, Hardeman R (2017) Police Brutality and Black Health: Setting the Agenda for Public Health Scholars. American Journal of Public Health 107(5): 662-665.

21. Leininger MM (1994) Transcultural nursing: concepts, theories, and practices. Greyden Press: Ohio. 